\title{
LAS TRADUCCIONES DEL TEATRO FRANCÉS (1700-1835). MÁS IMPRESOS ESPAÑOLES
}

\author{
Maria Jesús GARCía GARROSA \\ Germán VEGA GARCÍA-LUENGOS
}

La finalidad del presente repertorio es aportar más datos concretos sobre las dimensiones que el teatro francés adquirió en España durante el siglo XVIII y el primer tercio del XIX. Los contornos del notable fenómeno, en su significante repercusión editorial, quedaban mareados objetivamente para los estudiosos con el excelente catálogo de Francisco Lafarga, Las traducciones españolas del teatro francés (1700-1835). I: Bibliografía de impresos*, del que estas líneas pretenden ser un modesto complemento. Sus 674 entradas - más las 46 del apéndice del segundo volumen- se completan ahora con otras 72. En buena parte de los casos se trata de sueltas, forma habitual de difundir tanto el teatro novedoso como el que sigue las pautas forjadas en la anterior centuria.

La mayoría de las fichas vienen a engrosar el número de impresos de títulos ya conocidos; lo que, sin duda, contribuye a subrayar la importancia cuantitativa del influjo francés como conjunto. Asimismo, en ocasiones, las cifras particulares consiguen poner aún en mayor evidencia la especial atracción que algunas piezas tuvieron para libreros y lectores, como eco fiel de su presencia y aceptación en los escenarios. Así, por ejemplo, las tres nuevas ediciones que aquí se describen de $L a$

* Barcelona, Universidad, 1983. Recientemente ha aparecido un segundo volumen dedicado a recoger los testimonios manuscritos de aquellas piezas de las que al bibliógrafo no le consta su edición (Barcelona, Universidad, 1988). Incluye asimismo un apéndice de Enmiendas y adiciones al volumen I. A partir de ahora las referencias a las entradas de ambos libros se harán por su número de orden precedido de LAFARGA, I y LAFARGA, Il, según el caso. 
Andrómaca y de Misantropia y arrepentimiento, hacen que el número de las conocidas de cada una de estas dos obras llegue a ocho, situándolas entre las más solicitadas por el público. En este sentido, cabe destacar las cuatro nuevas impresiones localizadas de La mujer de dos maridos, que suben muy significativamente el índice de su cotización, situado en dos en el catálogo de Lafarga.

La importancia de algunas de las entradas no se circunscribe únicamente a este flanco cuantitativo, sino que su consideración puede deparar rendimientos ecdóticos y literarios. Es el caso de las correspondientes a ediciones con fechas anteriores o iguales a las hasta ahora reseñadas, que, en principio, pudieran permitir un acceso más adecuado a los textos. Así ocurre con Blnuca y Montasín, El colegio de Tonnington, La corona de laurel, El desertor, Eduardo y Federica, Los ilustres payos, Inesilla la de Pinto, La mujer de dos maridos, Romeo y Julieta, FI sarrificio de Ifigenia. Segunda parte, Semiramis, Un ministro, La urraca Ladrona.

Son cuatro las ediciones de piezas de las que no constaba su plasmación en letras de molde, aunque se reseñen en LAFARGA II diferentes testimonios manuscritos: La dama doctora, La prueba feliz, Tomás Juan en Londres y Un desafio o dos horas de favor.

En el peldaño más alto de interés se encuentran las ediciones de cinco nuevos textos: Angelo, tirano de Padua, Los árabes en las Galias, El barbero de Sevilla, Ester y La extranjera.

Los criterios cronológicos, idiomáticos, etc. que nos han guiado en la inclusión de los diferentes impresos pretenden acoplarse a los utilizados en el meneionado catálogo. También siguen su pauta el orden y disposición adoptados. Sin embargo, be ha modificado el sistema descriptivo, a fin de acompasarlo con el que se va imponiendo en los repertorios más recientes de fondos teatrales antiguos. Este sistema facilita la identificación de las diferentes ediciones de una misma pieza, ante las pistrechas semejanzas que a menudo presentan.

Los catálogos utilizados y aducidos en las distintas entradas, con mención de las claves empleadas, son los siguientes:

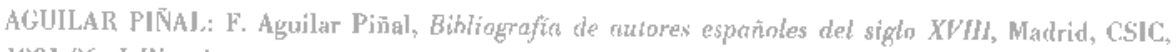
1981-86, I-JV vols.

AGULI, O Y COBO: M. Agulló y Cobo, tha colección de teatro do la Biblinteca Municipal de Madrid, Retrista de Literanura, XXXY-XXXVII (1969-1970), Revista de ta Biblioteca, Arehivo y Musen del Ayuntamiento rle Madrid, I-XII,

BAINTON: A. J. C. Bitinlon, 'Comedias stelkes' in Cambridge University Library: A Descriptive Catalogiate, Cambridge, The Universily Library, 1977.

BERGMAN-SZMUK: H. L. Bergman y S. E. Szmuk, A Catriogue of Comedias suelters in the New Yonk Public Library, Londres, Grant \& Cutler Ltd., 1980. 
BOYER: M. V. Boyer, The Texus Collection of Comedias sueltas. A Descriptive Bibliography. Relerence Publications in Latin American Studies, Boston, G. K. Hall and Co., 1978.

GREGG: K. C. Gregg, An Inder to the Spawish Theater Collection in the London Libury, Charlotesville, Bibliotera Siglo de Oro, 1984.

McKNIGHT-JONES: W. A. MeKnight y M. B. Jones, A Calatogtue of 'Camedias suctos' in the Librory of the Uniwersity of North Carotina, Chape] Hill, Univ. of North Carolina Library, 1965.

PAZ Y MEL1A: A. Paz y Melia, Papeles de Inquisicion. Catátogo y extracios, 2." ed., Madrid, 1947.

SULLIVAN-BLRSHAS: H. A. Sullivan y H. N. Berghas, The Wryne State University Collection of 'Compdias sueltas'. A Desriptive Bibliography, Detroit, Wayne State University Press, 1984.

ABATE DE L'EPEE, EL

1

EL ABATE/ DE LEPEE, Y SU DISCIPULO/ EL SORDO MUDO DE NACIMIENTO,/ CONDE DE HARANCOUR/ COMLUIA/ FN CINCO ACTOS, POR/ HONSEUR BOULLY, INDHVIDUO DE LA SOCIEDAD/ PHLOOTECHNCIA./ TRADUICIDA IIN CASTELIANO/ POR DON JUAN DE ESTRADA, Y D...LAASIFYZOS./ MADRIIY/ EN LA OIICINA DE D. BENITO GARCIA, Y COMPAÑIA./ AÑO DE 1800.

- Señor, dau tomprano? pro no oye... está

-cluído bien mi carrera.

FIN.

8. ${ }^{0}, \mathrm{~A}^{8} \mathrm{~B}^{\mathrm{H}} \mathrm{i}^{4}$, pp. |1-4,j-120. 'Texto al uni columna.

AUSTIN, Univernity of Texas, 862.08/T224/A2/v. 16 no.6 [Clr. BOYER, 46.1.21.

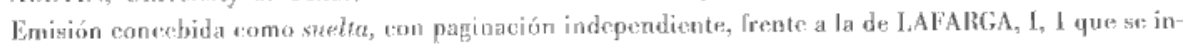
cluye en el Teatro Nuevo Espeñot, I, pp. 341-460.

ABATE L'EPEE, EL

2

EL ABATE L'DPEL/ Y LL ASISINO,/ O/ LA HUERYANA/DE BRUSELAS/ [filete]/ DRAMA DE ESPECTACUIO/ EN TRES ACTOS./ [adorno ovalado]/ VALENCIA: IMPRENTA DEL GIMENO. 1832./ SE hallari en su librering, frente ot Miguplete, te igutmente un gran suftido de romedias/ antiguas y morlernas y sametes.

-Dojad mi rocin en la puerta

$-[\ldots]$

8. ${ }^{4}, 1-5^{8} 6^{3}$, pp. $\left.\mid 1-3\right\rfloor-86$.

AUSTIN, University of Texas [Cti. BOYlR, 46.2].

ACAMENONN

3

AGAMISON./ TRAGEDIA/ TN CINCO ACTOS:/ ESCRYTA/ POR EI, CIUDADANO LUIS LEMERCIER,/ Y TRADUCIDA DISI FRANCES/ POR/ D. L. T./MADRI19/ EN LA OFICINA DE D. BENITO GARCIA, Y COMPAÑLA./ AÑO DE 1800,/ Se hallari en las Librerias de Quiroga, colle/ de Las Carretas, y de la Conrepción Geronimat.

-De tu solicitud y de tus viages.

-y á Minos pediré vuestro suplicio.

FIN.

8. ${ }^{\circ}$, A- $E^{4} \mathrm{~J}^{4}$, pp. [1-4.]-86. Texto a una columna.

AUSTIN, University of Texas, 862.08/T224/v.1 n. 3 [Cfr. BOYER, 136.3.1]. 


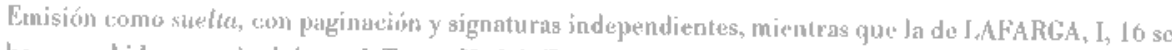
ha concebido para incluirse el Tumo II del Tentro Nuevo Español, 39l-476.

\section{ANDROMACA}

4.

N. 91, I/ TRAGEDIA HEROICA. LA ANDROMACA:/ POR OTRO TWTULO/ AL, AMOR DE MADRE/ NO HAY AFFCTO QUE LE IGUALI:

-Ismenia, ¿el Rey me ha visto?

- por ser su primer Tragedia.

FilN./ Barretonn: Por Juan Franriseo Piferrer, Impresor/ de S. R. M.; védeste en su J ibrería administrada/ por Juan Sellent.

$4^{4}, \mathrm{~A}^{-\mathrm{E}^{4}}, 40 \mathrm{pp}$.

SANTANDER, Biblioteca de Menéndez Pelayo, 414.

5

N. 23./ TRAGEDIA NUEVA./ LA ANDHOMACA./ POK OTRO TITULO./ AL AMOR DE MADRE/ NO HAY AFECTO QUT, LE IGUALL.

-Isincuia, iel Roy me ha visto?

-por sel su prituer Tragedia.

FIN./ Se hallará en la I,ibrería de Casimiro Razola calle de/ Aucha frente ú la Aduana Viejal y én İa do Quiroga, callef de la Conceepciurn.

$4{ }^{2}, \mathrm{~A}-\mathrm{E}^{2} \mathrm{~F}^{2}, 4.3 \mathrm{pp}$. En blanco la p. 4.4.

SANTANDER, Biblioteca de Menénde. Pelayo, 31809; (GHAPEL IIII.. Universily of Norll, Carolina, TAB 19,4 [CIr. MA:KNIGHT-JONLS, IIg].

6

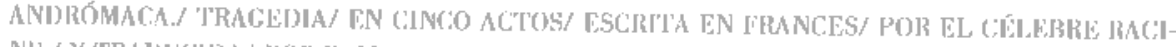

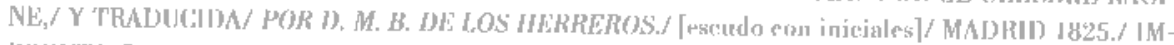
I'IRINTA LEL D. MICUEI, DE BURGOS.

Sí: má ingrata fortuna va á mudarse

-lnúliles scrán nuestros esfucrzos.

8.", a-ce $\mathrm{e}^{8}, 88$ pp. Texto a una crolumna.

SANTANIDER, Biblintera de Menéndez Pelaya, 32301.

ANGEI.O, TIRANO DE PADUA

7

ANGFLO, TIRANO DE PÁUUA./ Drama en tres jornarlas/ ESCRT'TO EN FRANCÉS/ por HA. Victor

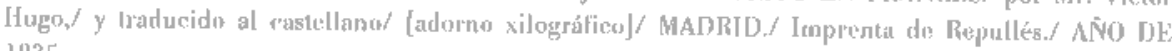
1835.

-Sí, monseñor, sois el magnifito podestá, èl ducño

-muesto. - Vivid. - Yo te bendigo.

FIN.

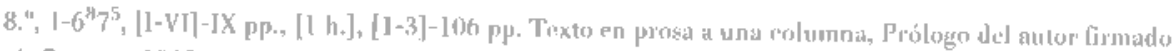
A 7 mayo 183.5.

MADRID, Biblivteca Nacional, T-8254.

ARABES LN LAS GALAS, LOS

\&

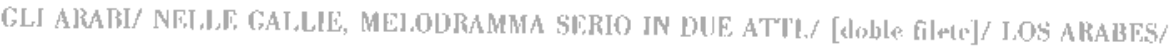


EN LAS GALIAS, MELODRAMMA SÉRIO EN DOS ACTOS./ QUE SE HA DE REPRESENTAR/ LN LOS TEATROS DE ESTA CORTE./ [figura tipográfica con instrumentos musicales]/ MADRID:/ IMPRENTA DE I. SANCHA./ [filete]/ 1829.

-Ahi! qual tremendo suono!

-Que horror! jamás se vió una escena mas/ lúgubre.

FIN DEL MELODRAMA.

8. ${ }^{\circ}, 1-4^{8} 5^{1}$, pp. [1-7]-65. En blanco la última pág. Texto a una columna. Verso italiano en el reverso de las hojas y prosa castellana en el recto.

AUSTIN, University of Texas, TF $24.13,862.08 / T 225 /$ ser.2/v.6 n. ${ }^{\circ} 3$ [Cfr. BOYER, 0.3, 0.3 A y $0.3 B$ ].

Adaptación de Luigi Romanelli de la novela Le renéget de D'Arlincourt.

AVISO A LOS C.ASADOS, EL

9

AVISO/A LOS CASADOS,/ COMEDIA EN TRES ACTOS,/ POR D. F. E. CASTRILLON./ REPRESENTADA EN EL TEATRO/ DE LA CALLE. DEL PRINCIPE/ DL AÑO DE 1807./ CON LICENCIA:/ EN LA OFICINA DE D. BENITO GARCIA Y COMPAÑ]A, AÑO DE 1808./ Se hallara en la libreria de Quiroga, tcalle de las Carretas.

-Llega usted precisamente

-y separa del acierto.

8. ${ }^{\circ},[\mathrm{A}]-\mathrm{F}^{B}$, pp. [1-4]-78. Ultima haja en blanco. Texto a una columna.

AUSTIN, University of Texas. [Cfr. BOYER, 44.2].

No consta ninguna edición de la pieza en LAFARGA. Copias manuscritas en II, 65 y 66 .

BARBERO DE SEVILLA, EL

10

EL BARBERO/ DE SEVILLA./ OPERA BUFA/ QUE SE HA DE REPRESENTAR POR LA/ COMPAÑIA ITALIANA EN EL TEATRO/DEL PRINCIPE DE ESTA/CORTE./ [banda]/ MADRID:/ Imprenta de la Minerva Española./ R. J. Fernández, 1822.

-Piano, pianissimo.

-voso-/ tros reinar.

8.", $1-6^{9} 7^{6}$, pp. [1-2]-107 [108].

Texto italiano en verso en el reverso de las hojas y español en prosa en el recto.

AUSTIN, University of Texas, 862.08/T224/A2/v.12 n. 6 [Cfr. BOYER, 0.9].

Adaptación de Le barbier de Sénille de Beaumarchais a cargo de Cesare Sterbini. Fs versión diferente a la consignada en LAFARGA, I, 60.

BLANCA Y MONTCASIN

11

BLANCA Y MONTCASIN, / O/ LOS VENECIANOS./ TRAGEDIA EN CINCO ACTOS./ ESCRITA EN FRANCES./POR EL CIUDADANO ARNAULT./ TRADUCIDA AL CASTELLANO./ POR DON TEODORO DE LA CALLE./ Representada en el featro de los Caños/det Peral en este presente año./ MADRID:/ EN I,A OFICINA DE CABALLERO./ AÑO DE MDCCCII./ Se hallará en el puesto de Sanchez, calle del Princi-t pe frente al Coliseo.

-Generoso extrangero, á quien se debe

-en medio de verdugos inhumanos.

FIN,

8.", A-F $\mathrm{F}^{8}, 96$ pp. Texto a una columna.

SANTANDER, Biblioteca de Menéndez Pelayo, 31599; AUSTIN, University of Texas, 862.08/T224/v.2 n." 7 [Cfr. BOYER, 22.1 y AGUILAR PINAAL, Il, 710]. 
12

N. 37./ BRITANICO./ TRAGEDLA EN PROSA/ EN CINCO ACTOS/TRADUCIDA DEL FRANCES/POR DON SATURIO IGUREN.

$$
\begin{aligned}
& \text { - ¿QUé esto [sic]? Quando Nerón està } \\
& \text {-sus majdades. }
\end{aligned}
$$

Fin./ Barcelona: Por Carliss Gibért y Tutó, Impresor y Librero.

$4{ }^{\circ}, A-D^{4},[1]-32$ pp.

SANTANDER, Bibliolewa de Menéndez Pelayo, 34176.

\section{BRETO}

1.3

Núm. I/ BRUTO/ TRAGEDIA DE M ${ }^{3}$, DE VOLTAIRE/ Tratucida del Frances en Lispanol./ POR R. GARCIA.

$$
\begin{aligned}
& \text { - Valerosos Romanos, } \\
& \text {-demos Gracias a los Dioses. }
\end{aligned}
$$

[En el centro de un adormo tipográfico lloral:] FINIS

4. ${ }^{\circ}, \mathbf{A}-\mathbf{E}^{4},[1]-39$ pp.

MADRID, Nacional, 'I'-4657 [Cfr. AGUILAR PIÑAL, IV, 447].

CARPANTERO DE LIVONLA, EL

14

Núm. 159. I/ COMEDIA NUEVA/ EL CARPINTEPO DE LIVONIA./EN TRES ACTOS.

-Al fin os veo Señorita; ya hu-

-Carpinte-f ro en una posada de Livonia.

FIN./ CON LICENCIA./ [doble filete]/Barcelona: En la Oficina de JUAN FRANCISCO PIFERRER,/ Impresor de S. M.; véndese en su librería administrada/ por Juan Sellent.

4. ${ }^{\circ}, A-C^{4} D^{2}, 1-28$ pp.

SANTANDER, Menéndez Pelayo, 34213.

CASADO POR FUERZA, EL

15

SAYNETE: FL CASADO POR F'UERZA.

- Márchate á casa, que yo.

-el perdon de nuestron yerros.

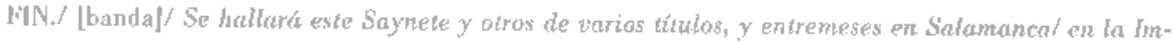
prentr de Celestino Harmel Rodriguez-Grande, Calle Serranos, numero 36.

4.,[]$^{4}$, pp. []]-8.

AUSTIN, University of Texas [Crr. BOYER, 39.6].

CASAMENTO DESIGUAL, EL

16

[p. 3:] EL CASAMIENTO DESIGLAL.

-Tudus lus que fueren tontos

-perdonen las faltas nuestras.

FIN./ En ol despracho de estat Imprenta yef hallarti surtido de varios tílos de Say-/ netes, Entremeses y Conedias.

4. ${ }^{\circ}$, pp. [1]-12. 
SANTANDER, Menéndez Pelayo, 30660.

El ejemplar reseñado carece de la primera hoja. Es una edición diferente de las dos catalogadas en LAFARGA, I, 107 y 108.

CELMIRA, LA

17

TRAGEDLA./ LA CELMIRA./ EN CINCO ACTOS./ TRADUCDA/ DEL FRANCES AL CASTELLANO./ CORREGHA Y ENMENDADA EN ESTA MMPRESION.

-NO me huyas, Ema querida,

-de mi hija en los sentimientos.

FIN,

$4 .^{\circ}, \mathrm{A}-\mathrm{D}^{4}, 31 \mathrm{pp}$. En blanco la última página.

SANTANDER, Biblioteca de Menéndez Pelayo, 30785.

18

N. 34. 1/TRAGEDIA./LA CELMIRA./EN CINCO ACTOS./TRADUCIDA DEL FRANCES./ CORREGI-

DA Y ENMENDADA EN ESTA SEGUNDA IMPRESION.

-NO me huyas, Ema querida,

- de mi hija en los sentimientos.

PIN./ Barcelona: En la Imprenta de Carlos Gibért y Tutó,/ Impresór y Librero.

4.", A-D $\mathrm{D}^{2}, 1-36$ pp. Papel azulado.

SANTANDLR, Biblioteca de Menéndez Pclayo, 34140(4); CHAPEI, HILL, University of North Carolina, TAB 30, 22 [C.fr. MCKNIGHT-JONES, 342].

COLEGIO DE TONNINGTON, EL

19

EL/ COLFGIO DE TONNINGTON/ $6 /$ LA EDUCANDA,/ DRAMA EN 6 ACTOS./ POR VICTOR DUCANGE,/ AUTOR DE LOS TREINTA AÑOS 0 LA VIDA/ DE UN JUGADOR, ETC./ Con liceneia/ BARCLLONA,/ IMPRLNTA DE A. BERGNES Y COMP./ CALLE DE ESCUDLLLERS, N. 13./ NOVIEMBRE 1831.

- Deberé todavia repetir estas palabras?...

-Ay de mít Victima infeliz.

FIN

16. ${ }^{\circ}$, pp. [1-7]-200. Texto en prosa a una columna. [P. 5; Sotete a la Sra. Carolina del Castillo, que interpreta el papel de Elena en el drama, firmado por J. L. D. M.

MADRID, Biblioteca nacional, T-11496.

CORONA DE LAUREL, LA

20

LA CORONA DE LAUREL, O/ LA FUERZA DE LAS LEYES/ DPAMA NUEVO/ EN CINCO ACTOS EN PROSA/ [banda]/[adorno oval]/ VALENCIA:/ IMPRENTA DE MUNOZ Y COMPAÑLA. 1821. -Venid conmigo; nada receleis.

-Mi hijo! Querido hijo mio!

FIN DEL DRAMA.

8. ${ }^{0}, 1-4^{8} 5^{1}$, pp. $[1-3]-71$ [72]. Texto en una columла.

AUSTIN, University of Texas, 862.08/T224/v.4 n. 1 [Cfr, BOYER, 0.26]. 
COMEDIA/ FAMOSA,/ INTITULADA:/ LA DAMA DOC'OORA, O LA/ THEOLOGIA CAYIA/ EN IA

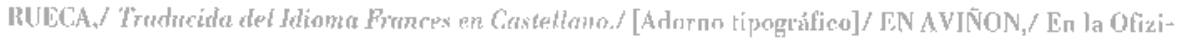
na de FliANCISCO GIRARDO,/ [doble filete]/ Con Lzencia de los Suppriores.

-FINEIR? Señora Angélica?

-Inqui-/ sizson acaba de llegar do Lispuīa.

Fin de lo Comedia.

8." pp. [1-2]-187.

MADRID, Biblioteca Nacional, T'-22,439. [Citr. PA7, Y MLIJ A , 27I].

No consta ninguna edioinn en LAFARGA. Para copiag manuberitas, ver II, 157 y 158.

DESERTOR, EL

22

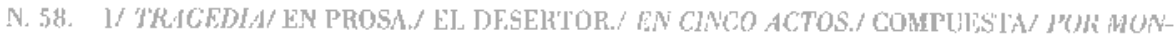
SHER MLRCJER./ TRADUCJDA DEL FRANCES AL ESPAÑOL.

-BlJenos estamos. i0 derventurado

-tra justa alegria.

HIN./ Barcelona: Tin la lmprenta de Carlns Gibert y Tuló,/ Impresur y Meroader de Libros.

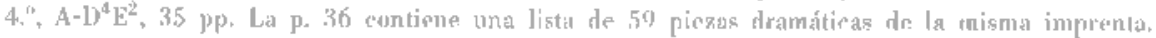
SANTANDER, Biblioteca do: Menéndez Pe]

\section{EDUARDO Y FEDERUAA}

23

EDUARDO Y FEDFRICA, COMTDIA NUEVA, HN PROSA, EN TRES ACTOS.

- Peste en la bodá, en los novios

-dv: Jos mortajes afligidor.

CON LIC:ENC:IA:/ SEVILLA, Imprentu de Cáto. Año de 1817./ [filete]/ En la misma Impsenua y Librervir

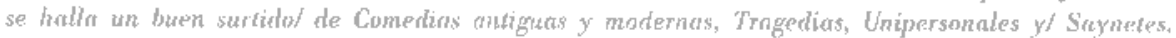
$4 .{ }^{\circ}, 1-3^{1}-[]^{\prime}, 25$ pp. Ullima página en blanco.

SANTANDER, Biblioteca de Menénder. Pelays, 188.

24.

EDUARIOO/ Y/ FEDERHCA./ COMEDIA EN TRES ACTOS./ EN PROSA. VALENCIA:/ IMPRENIA DL

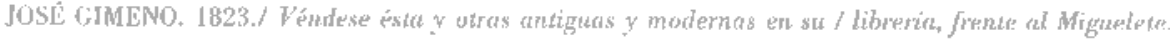

-Pestre en la bada, en los novios, y en

-mas de los morlales alligidos.

FIN.

8.", 1-3 $4^{2}{ }^{2}[]^{\prime}, 5.3 \mathrm{pp}$. Última página en blaneo.

NUEVA YORK, ['ublje Library, NPL p.v. 619 [Cfr. BFRGMAY-SZMUK, 408].

EMPERADOR ALBERTO I Y LA ADELINA, EL

25

1/ GOMEDIA NULVA./ EL EMPERADOR/ AT,BERTO I./ Y LA ADELINA./ PUESTA EN VFRSO, EXORNADA Y ARRECLADA/ A NUESTRO T'EATRO/ POR D. ANTONO WALLADARES DE SOTOMAYOR.

-Esta es la casa, Gerardo;

-y вe aplauda lo instruetivo.

FIN. [5.l, s.i., s.i.] 
4. ${ }^{\circ}, \mathrm{A}-\mathrm{D}^{4} \mathrm{E}^{\mathrm{I}}, 34 \mathrm{pp}$.

NUEVA YORK, Public Library, NPL p.v. 209 [Cfr. BERGMAN-SZMUK, 424]. DETROIT, Wayne State University, SD 6 [Cfr. SULLIVAN-BERSHAS, 352]. CHAPEL HILL, University of North Carolina, TAB 37,1 y $862,8 / \mathrm{T} 254 / 1,1$ (Cír. MoKNIGHT-JONES, 682].

ESPANOOL Y LA FRANCESA, EL

26

EL ESPAÑOL/ y la/ FRANCESA. COMEDIA EN UN SOLO ACTO./ MADRID: IMPRENTA QUE FUE

DE GARCIA./ AÑO DE 1831./ Se hallará en la librería de la viuda de Quiroga, calle de las Carretas; juntamente con un grarı sur-/ tido de comedias, tragedias, sainctes y demás pie-/ zas dramáticas.

-No hai nadie: bien puedo entrar

- pues las paces ya estau hechas.

FIN.

$8 .{ }^{\circ}, \mathbf{l}^{8} 4^{8}, 63 \mathrm{pp}$. La última página contiene una relación de títulos.

NUEVA YORK, Public Library, NPL p.v. 326 [Cfr. BERGMAN-SZMUK, 470].

ESPIGADERA, LA

27

N. 107. I/ COMEDLA NUEVA./ LA/ ESPIGADERA./ CORREGIDA Y ENMENDADA EN ESTA 2." IMPRESION.

-PAsa el tiempo tan velóz

-Con aplaudirla de veras.

FIN./ Barcelona: En la Imprenta de Carlos Gibert y Tutó,/ Impresor y Mercader de Libros. $4 .^{\circ}, \mathrm{A}-\mathrm{D}^{4} \mathrm{E}^{2}, 36 \mathrm{pp}$.

CAMBRIDGE, University Library, Hisp, 5.76 .25 (14) [C.fr. BAINTON, 321].

ESPLIN, EL

28

EL ESPLIN./ PIEZA EN UN ACTO:/ POR/ D. YICENTE RODRIGUEZ DE ARELLANO./ REPRESENTADA/ POR LA COMPAÑIA/DEL SEX̃OR EUSEBIO RIBERA./ [adorno tipográfico]/ CON LICEN-

CIA:/ En Valencia: Por Josef Lstevan, plaza de S. Agustin./ Año 1798.

-Querido Jacobo mio...

- ayer en la olra posada.

FIN./Se hallará en la Librería de Francisco Navarro, á/ los Hierros de la Lonja, junto con un completo/ surtido de Comediaz antiguas, Tragedias, Piezas/modernas, y una Coleccion de Saynetes/ de D. Ramon de la Cruz, sueltos/ y enquadernados.

$4 .^{\circ}$. sin sigs., $15 \mathrm{pp}$. Ultima página en blanco.

SANTANDER, Biblioteca de Menéndez Pelayo, 32915.

ESTER, LA

29

ESTER,/ DRAMA SACRO/ EN DOS ACTOS./ POR/ D. L. F, C./ REPRESENTADO EN EL TEATRO DE LOS CAÑOS DFL PERAL EN LA QUARESMA/ DLL AÑO DE 1803./ [adorno xilográfico]/ EN MADRID/ EN LA IMPRENTA DE SANCHA.

- Clemencia, ó Dios benefíco,

- porque en Dios fundada está.

FIN.

8. ${ }^{0}, \mathrm{~A}-\mathrm{D}^{8} \mathrm{E}^{7}$, pp. [1-2]-77. Ultima página en blanco. Texto a una columna.

MADRID, Biblioteca Nacional, T-21225. 
30

I.A STRANIERA, / MELODRAMMA/SERIO IN DUE, ATTI / [doble filete]/ LA EXI'RANGERA, MEI,ODRAMA SERIO EN DOS ACFOS, QLIE SE HA DE REPRESENTAR/ FN IOS TF.ATROS IT FSTA COR'T'E./[escudo con instrumentos musicales]/MADRID:/IMPRENTA DE I. SANCHA./[filete]/ DACIEMBRE DE 1830.

$$
\begin{aligned}
& \text { - Voga, viga, il vento tace, } \\
& \text {-ciejo, tanto estravio. }
\end{aligned}
$$

FIN DEL MELODRAMA.

8.,$\left[1-7 \mid-5^{\text {9 }}\right.$, pp. [1-7]-79. U]tima páginá en h] dn1 m. Texto a una columna. Verso ilalians en el revarso y tradución española en prosia en el recto de las hojas.

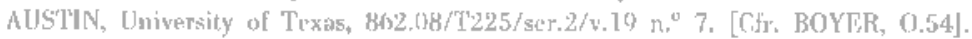

Fin el Argumento (pp. 3-4) se dice que el libretu-obra del poeta liomani- está basado en -una ingeniosa

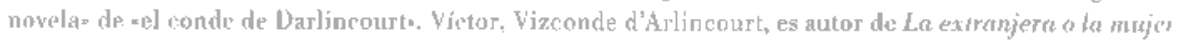
misterjosa (1825), nowela de la que se Lazaron on España repetidas ediciones: Valencia, 1830; Bdrceloпa, 1836; Barcelona, 1843; Barcelona, 1844; Valencia. 1847; Barcelona, 1844 (CCr. I. F. Montesinos: Intondercion a wata hisurin de la nowein en Fspeña, Madrid, Castalia, 1080, pp. 155-158).

31

LA/ ESTRANGFRA/ MELODRANA EY DOS ACTOS./TRADUCIDA AI FSPAÑOL/ CON ARREGLOA

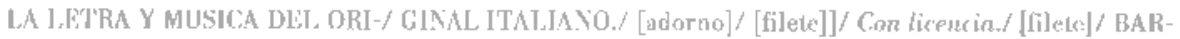
CFLONA: IMPRENTA DE D. RAMON INDAR./ CALIL DE ESCUDLLLERS N. ${ }^{\circ} 3 . / 1833$.

- Vurgi, vogna, en rielo claro

-Dies, perdona, tanto error.

I'IN.

8. $,[1]-3^{4}, \mathrm{pp} .[1-5]-47$. . Ultima página en blanco. Texto a una columna.

AGUSTIN Universily of 'lexis, 862.08/T224/v, 8 u." l. [CH. BOYER, 0.55]].

FLOSOOFO CASADO, HL

32

N. 29. I/ EL FILOSOFO CASABO;/ O ET, MARIDO/ AVERGONZADO DI SERLO./ EN CINCO AOTOS.

$$
\begin{aligned}
& \text {-EN este ratim estoy } \\
& -d e \text { inmensas telicidates. }
\end{aligned}
$$

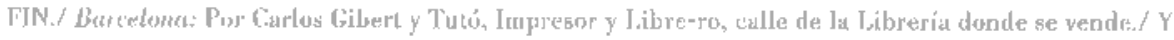
en Madrit en la de Manuel Quirogral calle de la/ Coneepeion, junto el barrio nuevo.

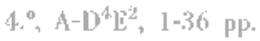

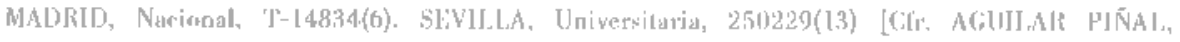
IV, 4017].

HIPOCRITA, EL

33

[Grubado de uma estena de la pieza// EL HIPOCRITA / TRADUCIDA AL ESPAÑOL/ por D. Joné Marchena/ [idoble filete]

-Anda, liejipal, más vives,

-Murho ha que ess metrescedoria.

4." pp. [303]-34.1. Consta al pie en [3. 341 - TOMO V.

SANTANDFR, Biblioteca de Menénder Pelayo, 32326. 
HUERFANO INGLES, EL

34

N. 9. I/ TRAGEDIA URBANA./ EL HUERFANO INGLES./ EN TRES ACTOS.

$-\mathrm{HE}$ concluido mi plan,

-En la eternida [sic] el pago.

FIN./ Barcelona: En la Imprenta de Carlos Gibert y Tutó/ Impresor y Librero, en la Libretería. 4.", A-C $\mathrm{C}^{-4}, 1-28 \mathrm{pp}$.

SANTANDER, Biblioteca de Menéndez Pelayo, 34140(5) y 34236.

ILUSTRES PAYOS, LOS

35

SAYNETE,/ INTITULADO/LOS LLUSTRES/ PAYOS,/ O/LOS PAYOS/ ILUSTRES,/ REPRESENTADO EN LOS TEATROS DE ESTA CORTE:/ PARA CATORCE PERSONAS./ [Grabado]/ CON LICENCIA/EN MADRID AÑo DE 1792./ Se hallarát en la Libreria de: Quiroga, calle de la Concepcion Gerónima.

-No hay dia mas alegre

- perdonad defectos tantos.

FIN./ En dicha Libreria de Quiroga [...] se hallara [...] y Tonadillas.

$4^{\circ}, a^{4}[]^{3},[1]-12$ pp.

SANTANDER, Biblioteca de Menéndez Pelayo, 783(22).

INESHLA LA DE PINTO

36

SAYNETE/ INTITULADO/INESILLA/ LA DE PINTO./ PARA SEIS PERSONAS./ [grabado con una carabela]/ CON LICENCIA/ EN CADIZ ANO DE 1800./ Se hallará en la imprenta de Quintana.

- Si estará por dicha en casa

-De todas las faltas nuestras.

FIN.

4.", $\sin$ sigs., $8 \mathrm{pp}$.

SANTANDER, Biblioteca de Menéndez Pelayo, 30657.

JUANITO Y JUANITA

37

SAYNETE,/ INTITULADO/JUANTO Y JUANITA,/ REPRESENTADO EN LOS TEATROS DE ESTA CORTE./ PARA NUEVE PERSONAS./ [grabado]/CON LICENCIA:/ EN MADRID: AÑo DE 1791./ Se hallará en la Librería de Quiroga, calle de la Concepcion Gerónima, / junto á la de Barrio-Nuevo: y asimismo otros de diferentes títulos: Co-medins cntiguas; Tragedias, y Comedias nuevas; Autos, Entremeses y/ Tonadillas.

-Negar á un vecino honrado

- logre perdón de sus fáltas.

FIN.

$4+^{\circ}, a^{4}[]^{2},[1]-12 \mathrm{pp}$.

SANTANDER, Biłlioteca de Menéndez Pelayo, $783(24)$.

JUVENTUDES DE ENRIQUE $Y$, LAS

38

LA GIOVENTU D'ENRICO V./ OSSIA/ LA BELLA TABERNARA./ MELODRAMMA BUFFO IN DUE, ATTl./ [doble filete]/ LAS JUVENTUDES DE ENRIQUE V./ O SEA/ LA BELLA TABERNARA./ MELODRAMA JOCOSO EN DOS ACTOS; QLE SE HA DE REPRESENTAR/ EN LOS T'EATROS DE ESTA CORTE./ [adorno tipográfico]/ MADRID:/ IMPRENTA DE I. SANCHA./ [filete]/ MAYO DE 1832. 
- hiche li giovamo

-tu corazon los latidos de gozo y amor.

FIN DEI, MELODRAMA.

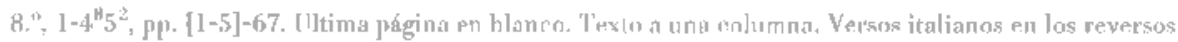
de las hojas y traducción espatoola en рговн en los rectos.

AUSTIN, University of Texas, 862,08/T225/5er.2/4.36 n." 4. [Ch. BOYER, 0. 76].

\section{MARIA ESTUARDA}

39

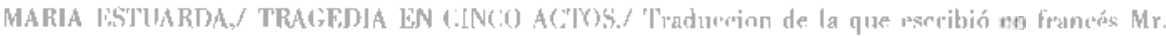

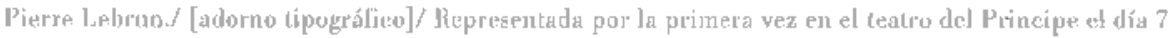
do. Noviembre de 1828. (*)

-iÁh! Por piedad, á mis humildes ruegos

4. pp. [1 19]-100. Desgajada de un tomo sin identificas', En nola al pie de la primera página se dice que la tradurción tue iniriada por Fentura de ta Kega.

SANTANI)ER, Bibliotpea de Menérdez Pelayo, 32342.

MARDO) DE SU HHA, EI

40)

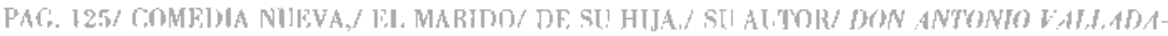

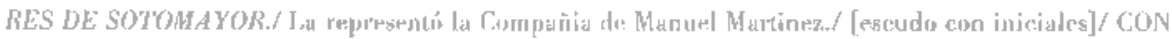

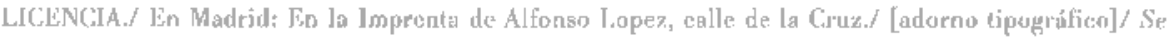

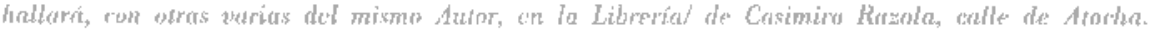

- Nadie en coda pstal $\mathrm{cs} / \mathrm{t}$ tancia.

-hoy tus aplausos merezca.

$[\mathrm{IIN}$ N

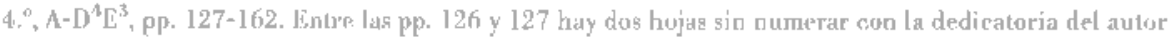
a D. Josela de Bempica, Fechada en Madrid, el 24 de agosto de 1736.

SANTANDER, Biblintera de Menéndez Polayo, 267.

El cjemplar santanderino presentá dos firales, Li grueso de la comedia y el printero de ellos pertenecen a la misema edirión reseñada por J AFARGA (BIT 45126). Sin embargo, a continuarión hay otras tres hojas,

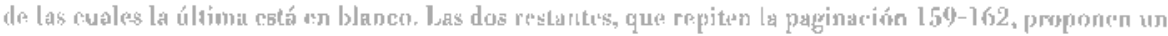
remale diferente para la pieza, alterando el orden do las tiradas de versos en varias orasioncs.

\section{MATHDE DE SHABRAN}

41

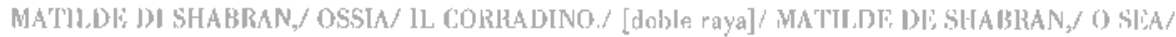

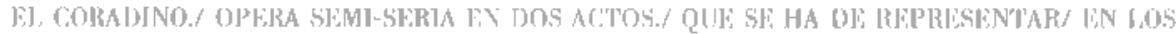

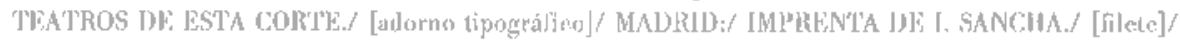
JUIOIO IJE 1831 .

-Zitti: nessum qui v'è.

- por ti.

TIN DEL MELOIDRAMA.

8., $1-6^{\mathrm{B}}$, pp. [1-7]-95. IJlima página en blanco. T'axto a una columa. Versas italianos en las reversos da las hojas y su traducción en prosa custelluná en los rectos.

AlJSIIN, Iniversity of T'exas, B62.08/T225/ser.2/y.42 n." 2. [CIr, BOYliR, 0.49].

Se crata de una edición de la versión de Jatopo Ferretli de la Eatphrosine de Benoit Hofirnan. Con el pri-

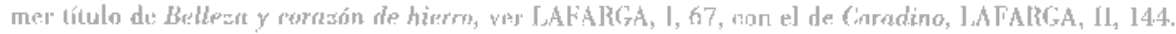


42

1/ DRAMA NUEVO/ EN TRES ACTOS/ LAS MINAS DE POLONIA,/ TRADUCIDO/ POR D. MARIA DE GASCA Y MEDRANO.

-En fin, estás ya de vuelta?

- corone el merecimiento.

Barrelona: En la Imprenta de Agustin Roca, calle de La Libreteria.

4. ${ }^{\circ}, \mathrm{A}-\mathrm{D}^{4}, 32 \mathrm{pp}$.

NUEVA YORK, Public Library, NPI p.v. 272 [Cfr. BERGMAN-SZMUK, 784].

MISANTROPIA DESVANECIDA, LA

43

Núm. 113. I/ LA MISANTROPIA/ DESVANECIDA./ DRAMA EN UN ACTO,/ ESCRITO EN ALEMAN/ POR AUGUSTO KOTZEBOE, EN CONTINUACION AL DRAMA INTITLLADO/LA MISANTROPIA/Y EL ARREPENTIMIENTO/ DEL MISMO AUTOR.

-Barre [sic], limpia, pobre desgracia-

-tros brazos.

Se abrazan los tres, y cae el telon.

FIN./ CON LICENCIA./ Barcelonn: POR JUAN FRANCISCO PIFERRLR, Impresor de S. M.; véndese/ en su Librería, administrada por Juan Sellent.

$4 .^{\circ}, \mathrm{A}^{4} \mathrm{~A} 2^{4} \mathrm{~A} 3^{2}$, pp. $1-20$.

SANTANDER, Menéndez Pelayo, 34242; AUSTIN, University of Texas [Cfr. BOYER, 0.94].

\section{MISANTROPIA Y ARREPENTIMIENTO}

44

N. 320. MISANTROPLA Y ARREPENTIMIENTO. DRAMA EN TRES ACTOS.

-AMigo Pelers, señora

-abraza á tu esposo:: -Eulatia. Oh cielo!

Los dos se throjan... cae el telon.

[Valencia, Joseph de Orga, Madrid, Quiroga, 1801].

4." A- ${ }^{4}$, pp. 1-32.

CHAPEL HILL, University of North Carolina, TAB 35,10,c.2 [Cfr. McKNIGHT, 1201].

45

N. 320. Pág. V/MISANTROPIA/ Y ARREPENTIMIENTO./ DRAMA EN TRES ACTOS,/ARREGLADO A NUESTRO TEATRO.

- AMigo Peters, señora

-abraza á tu esposo:: - Eulalia. O cielo!

Los dos se arrojan... rae el telón.

FIN./ CON LICENCIA: EN VALENCIA: en la Imprenta de Joseph/ de Orga, donde se hallará, y en Madrid en la Iibreria/ de Quiroga, calle de las Carretas./ Año de 1801.

$4 .^{\circ}, \mathrm{A}^{-\mathrm{E}^{4}}$, pp. $1-34$.

SANTANDER, Menéndez Pelayo, 34.245.

46

Núm. 120. MISANTROPIA Y ARREPENTTMILNTO. DRAMA EN TRES ACTOS. iCuán cruel es la venganza de la ultrajada virtud!

-Amigo Peters, Señora

-abraza á tu esposo... Eul. Oh cielo! 
Les dos the wrojorn... rap at telon.

[Valencia, Ildefonso Mompié, 1822].

$4,0,1-4^{4}, p p,[l]-32$.

CHAPFI. HII, Iniversity of North Carolina, TA 18,8 [Cfr. MCKNIGHT-JONF,, 1203].

MUJER DE DOS HARDOS, LA

4.7

V/ COMEDIA NUEVA./ LA MUCER DE DOS MARIDOS./ EN TRES ACTOS./ POR D. V. R. D. A.

-Atencion á lo que mando:

$-y$ mis volos or mimplierun.

Se hallará en la Libresia de la Viuda de Quirogá, calle de Jas Carretas número 9, con cuantas Comealias antiguas y modernas, 'Tragedias, Autos sacramen Lales, Saynetes y Linipersonales se han imfreso hasta esta época.

$4 ., A \cdot D^{4} E^{2}, 30 \mathrm{pp}$.

(CHAPEL HILL, Liniversily of North Carolina, I'A 22,7 [Clr. MoKNIGHT-JONES, 1239]. DETROTT, Wayne Slate [niversily, CV 39 y SD 12 [Cfr. SULLIVAN-BFRSHAS, 664].

48

Núrn. 161. I/ COMEDIA NUEVA./ JA MUGER DE DOS MARIDOS./ EN THES ACTOS./ POR U. V.R. D. $A$.

-Atención á lo que mando:

-y mis votos se vimpliéran.

FIN. CON LICENCLA./ Barchlona: En la Oficina de JUAN FRANCTSCO PIFElRRER,/ Impresor de S. M.; véralese en su librería alduninistrada/ por. Juan Scllent.

$4 .^{\circ}, \mathrm{A}-\Gamma^{4} \mathrm{E}^{-2}, 35 \mathrm{pp}$. Ulíma página en blanco.

NUEVA YORK, Public Lihrary, NPI p.y. 400) [Chr. BLRGMAY-SZMUK, 810]. LONDRES, London Library, pp. 954-2 [Cir. GREGG, 956].

4.9

Núm. IV. IA MUGER DE DOS MARIDOS. COMEDIA NUEVA EN TRISS ACTOS. POR D. V. R. D. A. -Atencion á lo que mardo:

-y mis votos se cumplieron.

[Valencia, Jasé Gimeno, 18237.

4.", 1-4 $4^{4}$, p. $\mathrm{s} .[1]-32$.

CHAPET, HILL, University of North Carolina, TAB 32,20 [Cf:, MeKNIGHT-JONES, 1240].

50

NÚm. 5./ COMLUIA NUFYA./ LA MLCELR DE DOS MARIJUS./ LN TRES ACTOS./ POR D. V.R. D.A. -Atenciour á Jo que mando:

-y mis vistus se eumplieron

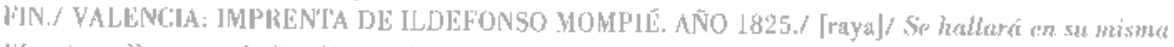

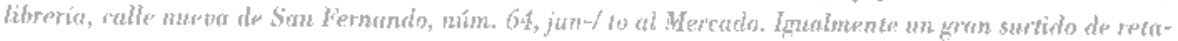
seria, ostampes pindolos y/ negras, comedian, sainetos y unipersonales.

$4 ., \quad 1-4)^{\prime}, 32 \mathrm{pD}$,

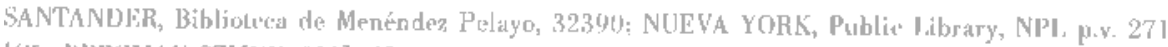
[1:Jr. BERCMAN-SZMUK, 811]; CHAPEL HILL, Liniversity of North Carolina, CTAE 21,5 y TAB 32,21 [Cfr. MCKNIGHT-JONES, 1241 y 1241a]. ALSTIN, Cniversity of Texas [Cfr. BOYER, 122,60]. 
NOVELERO, EL

51

SAYNETE,/ INTITULADO/ EL NOVELERO,/ REPRESENTADO EN LOS TEATROS DE ESTA CORTE./ PARA TRECE PERSONAS./ [grabado]/ CON LICENCIA:/ EN MADRID: ANOO DE 1791./ Se hallari en la Librerta de Quiroga, calle de la Concepcion Gerómima, jinto a ta de Barro-Nuevo; y asimismo otros de diferentes títulos: Co-t medias antiguas; Tragediax, y Camerlias nuever; Autos, Entrmeses y To-/nadillas.

- Hay hombres en la Corte

-el indulto suplicamos.

FIN.

4. ${ }^{\circ}, a^{4} b^{2},[1]-12$ pp.

SANTANDER, Biblioteca de Menéndez Pelayo, 783(30).

OTELO

52

N. ${ }^{\circ}$ 91./OTELO,/ O EL MORO DE VENECIA./ TRAgEDIA EN CINCO ACTOS,/ TRADUCIDA DEI.

FRANCES/ POR/ L. A. C. A. L. L. E.

-llustres y gloriosos Senadores,

-te volveré á estrechar... muero. Todos. O desgracia!...

[Valencia: Domingo y Mompié, 1821]

$4 .{ }^{\circ}, 1-3^{4} 4^{2},[1]-28$ pp.

LONDRES, London Library, p. 959-20 [GREGG, 1082]; DETROIT, Wayne State University, SD 13 [Cfr. SULLIVAN-BERSHAS, 730]; CHAPEL HILL, University of North Carolina, TA 12, 15 (Cfr. McK-

NIGHT-JONES, 1363].

\section{PABLO Y VIRGINIA}

53

PABLO Y VIRGINIA./ DRAMA I'ASTORAI. FN TRES ACTOS./ SACADO IIE LA HISTORIA/ QUE ESCRIBIO EN FRANCES/ SANTIAGO BERNARDINO ENHIQUE DE SAINT-PIERRE./ PUESTA EN VERSO/ Y ACOMODADA AL TEATRO ESPANOL/ POR DON JUAN FRANCISCO PASTOR.

[At pic de la p. 1:] Se hallará en la librería de la Viuda de Quiroga, calle de las Carretas, con un gran surtido de comedias, tragedias, sainetes y demas piezas dramáticas.

-Olvida el atiolo, querida:

-gozad siempre, gozad.

$44^{\circ}, \mathrm{A}-\mathrm{D}^{4} \mathrm{E}^{2}$; pp. $[1-2]-4,0$.

DETROIT, Wayne State Univerbity, SD 14. [Cfr. SULLIVAN-BERSHAS, 732]; CHAPEL, HILL, University of North Carolina, TE 424,5 y TA 21,9 [Cfr. McKNIGHT-JONES, 1365 y 1365 a].

PAYOS HECHIZADOS, LOS

54

SAYNETE./ LOS PAYOS HECHIZADOS.

-Al sonsonito

-Logre perdon de sus yerros.

FIN./ / En el despacho de esta Imprenut se/ hallari surito de varios títulos de Say-/ netes, Entremeses y Comedias.

4. ${ }^{\circ}, \mathrm{A}^{4} \mathrm{~B}^{2}$, pp. $[1]-12$.

SANTANDER, Menéndez Pelayo, 30662.

El ejemplar reseñado está falto de portada. Pertenecc a una edición diferente a las tres reseñadas en LAFARGA, 1, 494-496. 
(7) SAINETE NUEVO TITULADO LOS PAYOS HECHIZADOS, JUANTTO Y JUANITA.

- Al sonsito

-Logre perdon de sus yerros.

[Madrid, Marés y Cimpañín, 1865].

4.". sin sigs., PP. [1]-8.

CHAPEI, HIL, University of North Carolina, TAB 78,12 [Cfr. MeKNICHT-JONFS, 1404],

PRUEBA CAPRICHOSA, LA

56

IA PRIBIBA CAPRICHOSA./ COMEDLA/ EN DOS ACTOS, IMPRESA EN INGLES SIN LL NOMBRE/ DRI, AUTOR:/ TRADUCIDA EN FRANCES/ POR MADAMA RCCORONF/ Y TEL FRANCES EN CASTRI.LANO/ POR/ DON PRANCISCO DE PAULA NARANJO/ MADRID/ EN LA OFICINA DE D. BENITO GARCL, Y COMPAÑIA. A AÑO DE 1801./ Se hallará en los Librerís de Quiroga, calle/ de los Carretas y de la Concoprion Gernimima.

- La recomendacion de mi hermano es muy

-in-/ vierno!

FIN.

8. ${ }^{u}, A-C^{B} D^{6,}$, pp. [1-4]-59. Ultima página en blanco. Textu a una columná,

ALSTIN, University of Texas, 862.08/T224/v.14 n." 6 [Cir. BOYER, 105.1.2].

Emision como suelka, tom paginación y signaturas indeperdicntes; mientras que la de LAFARGA, I, 529 se ha conebido para incluirse en el Tomo VI del Teatro Nuevo Espeñol, pp. 113-171.

PRUEBA PELIZ, LA

57

I.A PliUEBA FELIZ,/ COMEDIA LA UN ACTO./ [vergos]

-Vaya, Señora, que está

- ipara qué hemos de cansarnos?

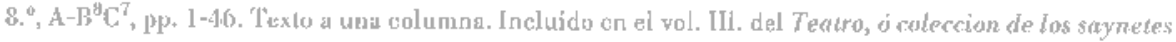

y dersas obras dramatiras de D. Remón de ta Criz y Cono, entre Los Arrades Larisio, Madrid, Imprenta Real, 1787.

No consta impreso en LAliARGA. Ina topia manuserita en Il, 4i38.

ROMLO Y NULFTA

58

Núm. 5. I/ ROMEO Y JULETA./ 'I'RAGLDIA WN CINCO ACTOS, TRADUCIDA DEL FIUNCLS. -NO tronas, nu, ¿Qué jimprs-

-ie Moutegon y rese el twhon.

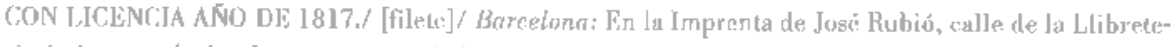

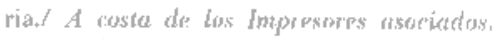

4. ${ }^{\circ}, C^{-4}, 24$ pp.

SANTANDER, Biblioleca de Menéndez Pelaya, 30732.

SACKHICIO DL HTGENA, EL

59

Pag. I, COMEDIA NVLVA./ EL SACRIFICIO/ DE ELIGENIA./ DF CINCO JORNADAS./ DE DON JOSEPH DE CANILARES.

- Viva Agamenon; y Troya 
-con esta [Bic] os ha divertido.

FIN./ CON IICENCIA. Barcelona: En la $1 \mathrm{~m}^{-/}$prenta de PFDRO ESCUDèr.

$4 .^{\circ}, \mathrm{A}-\mathrm{C}^{4} \mathrm{D}^{2}, 28 \mathrm{pp}$.

SANTANDER, Biblioteca de Menéndez Pelayo, 33644.

60

N. 9. I/ TRAGEDIA./ EL SACRIFICIO/ DE YFIGENIA./ DE DON JOSEPH DE CANIZARES.

-VIva Agamenon; y Troya

-ù dos palmadas, ò un vitor.

FIN./ Barcelona: En la Imprenta de CARLOS GIBERT y TUTo, Impresor, y Librero.

$4 .^{\circ}, \mathrm{A}-\mathrm{B}^{4} \mathrm{D}^{2}, 27 \mathrm{pp}$. Ultima página en blaneo.

SANTANDER, Biblioteca de Menéndez Pelayo, 34.182.

SACRIFICIO DE IFIGENIA, EL. SEGUNDA PARTE

61

N. 26 1/ TRAGEDIA./ EL SACRIFICIO/ DE IFIGENIA./ SEGUNDA PARTE.

-VIvan Toab, y Tomisis

-no imita en los aciertos la primera.

FIN./ Burcelona: Por Cartos Gibert, y 'Tutó Impresar, y Librero.

$4 .^{\circ}, \mathrm{A}-\mathrm{E}^{4} \mathrm{~F}^{2}, 44 \mathrm{PP}$.

SANTANDER, Biblioteca de Menéndez Pelayn, 34182(2).

\section{SEMIRAMIS}

62

N. 45./ TRAGEDIA FN UN ACTO:/ SEMIRAMIS./ POR D. G. Z. Y Z./ PARA CINCO PERSONAS.

Crimen abominable, cómo afliges

-reverenciamos los supremos juicios.

FIN./ VALENCIA:/ EN LA IMPRENTA DE ESTLVAN,/ AÑO 1813./ [filete]/ Se hrallará en la misma imprenta, frente al horno de Salicofres; y asimismo un/gran surtido de Comedias antiguas y modernas, Tragedias, Saynetes yf Unipersonales.

$4 . \%$ sin sigs., $16 \mathrm{pp}$. Texto a una columna.

SANTANDER, Biblioteca de Menéndez Pelayo, 32217.

SITIO DE CALES, EL

63

EL SITIO DE CALES:/ COMEDIA HEROICA/ EN TRES ACTOS./ Representada/ Por la Compañia de Manuel Martinez/ Ln el año de 1790.

Se hallará esta Comedia con la de Christoval Colon y la de el Hombre Agradecido en la Oficina del Diario, Plazuela de Zelenque, y en sus puestos Puerta del Sol y frente de Santo Tomás, en la Librería de Manuel Quiróga, calle de la Concepcion Gerónima, y en el Puegto de Manuel del Cerro, calle de Alcalá. $\Lambda$ dos reales.

$4 . ", 34 \mathrm{pp}$.

DETROIT, Wayne State University, SD 17 [Cfr. SULLIVAN-BERSHAS, 894].

SOLTERON Y SU CRIADA, EL

64

EL SOLTERON/Y SU CRIADA./ COMEDIA EN TRES ACTOS./FORMADA/SOBRE LA QUE ESCRIBIO EN FRANCES/ EL CIUDADANO COLLIN D'HARLEVILLE./ POR/ D. T. G. S./ MADRID/ EN LA 


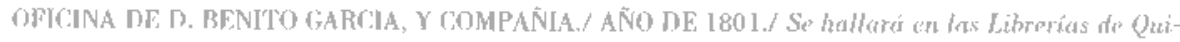
soga, onllo/ do las Cometas y de in Contepcion Gemónima.

- Ya está vestido. Arreglémos,

- la corrupcion busca en ella!

IIIN,

8." A-H ${ }^{8} 1^{4}$, pp. [1-60]-136. T'exto a una columna.

AUSTIN, Universily of T'exas, 362.08/T223/4.16 n." 1 y 862.08/T224/A2/v.9 n." 6 [CHr. BOYER, 62.1.2 y $62.1 .2 \AA]$.

Emisión comebida como suelta, con paginatión y signaturas imdependiontes, frente a la de LAFARGA, I, 580, que se iucluye en el Teatro Narevo Espontiol, Ill, pp. 457-592.

SORDO EN LA POSADA, EL.

65

Núm. 19./ DRAMA EN DOS ACTOS,/ INTITUIIDO:/ li, SORDO/ EN LA POSADA. TRADUCIDO

[III, FIANGIS./ POIT D. J. F. C.

-HOmbre, qué impariente es-

-del placer que dislrutamus.

[Valencia: [ldefonso Mompié; Miguel Domingo, 1816]

$4{ }^{\circ}, 1-3^{\prime}, 22$ pp. Lin las tos últimas páginas hay una lista de piezas.

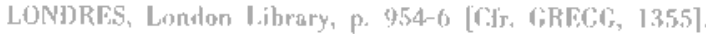

61

Núm. 19. I/ DURAMA EN DOS ACTOS, INTTTUTIADO: EL SURDO/ EN LA POSADA./ THADUCIDO DII. LIRANCLS/ POR D. F. E.C.

-Hombre, qué impaciente es-

-del placer que disfrutamos.

FIN./CON I.ICENCIA:/ VAIENCIA: EN I A IMPIRENTA DE ILDEFONSO MOMPIË/ Año 1816,/doble

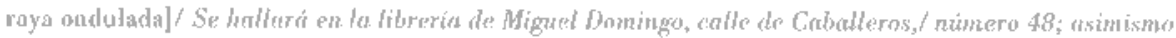

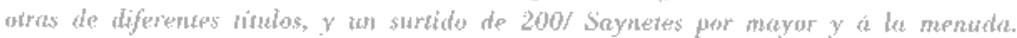

4.", ]-3, pp, 1-22. Lisla de piezas on la última hoja.

AUSTIN, Iniversily of T'Pxas: dos ejemplares [Cfr. Boyer, $44,9,2$ y 4,4.4.2A].

Sitivis, Fi.

66

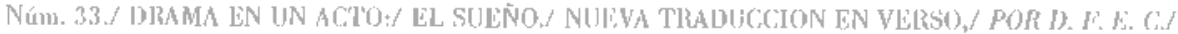
PARA CINCO PERSONAS.

- Señorila, pstá usted tristo

- 1 que esto no sra un supinte.

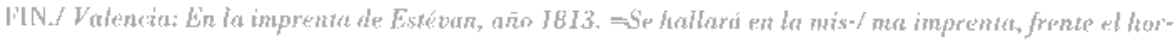

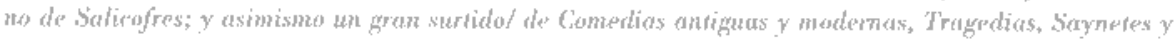
Unipersenales

$4 . "$. $\sin$ sigs., 16 pp.

NIIVYA YORK, Public Library, NPL p.e. 330 [Cfr. BERGMAN-SZMUK, I080].

TOMAS JUAN EN LONDRES, O FI, EXPOSIT

67

TOMAS JUAN/ ISN IONDRES, O/ LL ESPOSTTO,/ COMLDIA EN CINGO ACTOS/ SACADA DE LA NOVELA DE FITIJING, POR M. DLS/ FORGES; ARRFGIAIHA AL TJATRO ESI'AÑOI, Y/ TRADU- 
CIDA EN BERSO/ POR ANDRES PRIETO./[grabado con iniciales]/ BRUSELAS,/ IMPRENTA DE FRANCISCO-JOSE HUBLOU./[filete]/ 1825.

- En berdad señor patricio,

$-y$ de la birtud mi Alma.

FIN./ [Fe de erratas].

$8 .^{\circ}, 1-7^{6} 8^{2}$, pp. [1-5]-112.

SANTANDER, Biblioteca de Menéndez Pelayo, 32.246.

No consta como impresa en LAFARGA. Sí figura una copia manuscrita posterior en II, 504.

UN DESAFIO, O DOS HORAS DE FAVOR

68

$\left[\begin{array}{lll}\ldots & \ldots & \ldots\end{array}\right]$

-Se me ha respondido que cl lord canciller sigue

-remordimientos y una eterna separación.

[p. 42:] FIN DII. DRAMA./ / [p. 43:] Se hallara en Madrid en tas librerías de/Escamilla, calle de Carretus, y de Cuesta, frente á las Covachuelas.

$8 .{ }^{0},[1]^{7} 2^{B} 3^{4} *^{2},[1-3]-42$. Ejemplar mútilo de portada que ha sido sustituida por una hoja en la que se ha escrito a mano: - Un desafío/ o/ Das horas de favor./ Drama/ en/Tres actos/por Don Mariano Larra/(padre). AUSTIN, University of Texas [Cfr. BOYER, 79.4].

No figura como impresa en LAFARGA, que recoge un testimonio manuscrito en II, 522.

UN MINISTRO

69

UN MINISTRO!/ COMEDIA EN UN ACTO./ por Don Ventura de la Vega./ REPIRESENTADA/ en el Teatro del Principe el dia 10 de actubre/ de 1834./ MADRID: IMPRENTA DI, I. SANCHA./ Calle de la Concepcion./ 1834 .

-Jesús! el Señor D. Federico Mendoza por aqui...!

-Partido de oposicion.

8. ${ }^{\circ}$, pp. [1-5]-39. Texto en prosa a una columna.

MADRID, Biblioteca Nacional, T-10568.

URRACA LADRONA, I.A

70

LA GAZZA LADRA./ MELODRAMA/ EN DOS ACTOS, QUE SE HA DE REPRESENTAR EN/ FSTA CORTE POR LA COMPAÑIA/ ITALIANA EN EL PRESENTE AÑO/ DE 1822./ [banda]/ MADRID:/ IMPRENTA DE LA MiNERya ESPAÑOLA, A cargo de dun $J$. Fernandez.

-Oh che giorno forturiato!

-cias de un lardo arrepentimiento.

FIN DEL DRAMA.

8. ${ }^{\circ}, 1-7^{8} 8^{6}$, pp. [1-2]-123. Ultima página en blanco. Texto a una columna. Texto en verso y en italiano en el reverso de las hojas y en prosa castellana en el recto.

SANTANDER, Biblioteca de Menéndez Pelayo, 33381; AUSTIN, University of Texas, 862.08/T224/A2/ v. 2 n. ${ }^{\circ} 6$ [Cfr. BOYER, 0. 61].

Ver LAFARGA, I, 633.

VIAJES DEL EMPERADOR SEGISMUNDO, LOS

71

COMEDIA EN PROSA./ LOS VIAJES/ DEL EMPERADOR SEGISMUNDO,/ O/ EL, ESCULTOR,/ Y EL CIEGO./ EN QUATRO ACTOS./ TRADUCIDA POR DON DOMINGO BOTTI. 
- ¿Que significa aquella gente

-el caracter de la verdadera nobleza.

FIN./ BARCELONA./ Con Licencia. En la Imprenta del Diario.

$4 .^{\circ}, \mathrm{A}-[]^{4}$, pp. $[1-3]-29$. Ultima página en blanco. Faltan las pp. 25 y 26.

AUSTIN, University of Texas, TE XV.8. [CIr. BOYER, 16.1.1].

Diferente edición de la réел̀ada en LAFARGA, I, 644.

VIEJA Y LOS DOS CALAVERAS, LA

72

LA VIEJA, / Y LOS DOS CALAVERAS./ PIEZA NUEVA/ EN UN ACTO EN PROSA./ [adorno tipográfico owal]/VALENCIA: IMPRENTA DE GIMENO, 1823./ [filete]/Véndese en su librería, frente al Miguelele.t como tambien un gran surtido de romedios/ antiguas y modernas, tragedias y sainetes.

- Ya son las ocho, y Antonio no parece:

-que-/ rido padre.

FIN:

$8 .{ }^{\circ}, 1-2^{\mathrm{H}}$, pp. [1-3]-32. Texto a una columna.

ALSTIN, University of Texas. [CIr. BOYFR, 0,140.2].

Aurnque similar al la resentada por LAFARGA, I, 648, es tipográficamente distinta. 